\title{
Dynamic Response of Non-Uniform Rayleigh Beam Subjected to Harmonically Varying Moving Load
}

\author{
Jimoh, A. ${ }^{1}$, Ajoge, E. O. ${ }^{2}$ \\ ${ }^{1}$ Department of Mathematical Sciences Kogi State University, Anyigba, Nigeria \\ ${ }^{2}$ Centre for Energy Research and Development Obafemi Awolowo University, Ile-Ife, Nigeria
}

How to cite this paper: Jimoh, A. \& A joge, E. O. (2018) Dynamic Response of Non-Uniform Rayleigh Beam Subjected to Harmonically Varying Moving Load. Journal of Applied Mathematics and Computation, 2(8), 345-356.

http://dx.doi.org/10.26855/ja mc.2018.08.004 *Corresponding author: Jimoh, A., Department of Mathematical Sciences Kogi State University, Anyigba, Nigeria Email: jimad2007@g mail.com

\begin{abstract}
This paper investigates the effect of axial force and rotatory inertial on the dynamic motion of non-uniform Rayleigh beam resting on Pasternak foundation transverseby harmonically varying magnitude moving loads. The versatile Galerkin'smethod and the integral transform techniques were employed to treat the fourth order partial differential equation governing the motion of the vibrating system. Analytical solution was obtained for the transverse displacement response of the non-uniform Rayleigh beam. Analytical and numerical results show that as the values of axial force $(N)$ and rotatory inertial $\left(R_{0}\right)$ increases the deflection profile of the non-uniform Rayleigh beam decreases. It is also found that as the values of the other structural parameter such as shear module $(\mathrm{G})$, foundation modulus $(\mathrm{K})$ and damping coefficient $(\varepsilon)$ increases lead to decreases in the deflection profile of the beam. Finally, it is observed that the effect of rotatory inertia is significant compared to that of the axial force.
\end{abstract}

\section{Keywords}

non-uniform beam, axial force, rotatory inertia, Pasternak foundation, foundation modulus, damping coefficient.

\section{Introduction.}

The investigation of the dynamic response of elastic structures carrying one or more travelling loads is very important in Engineering and applied Mathematics as applications relate for example, to the analysis and design of highway, railway bridges, cable-railroads and the likes. Generally, emphasis is placed on the dynamics of the elastic structural members rather than on that of the moving loads. Common example of elastic structureincludes beams, plates and shells while travelling loads include moving trains, tracks, cars, bicycle, crane etc. However, studies on beam problems have largely been restricted to the case when beam structure is uniform. In particular, works on non-uniform Rayleigh beam is still an open problem in the field of science and engineering.

In non-uniform beam, the mass per unit length of the beam and flexural rigidity of the beam are not constant but becomes certain function of the spatial coordinate $x$ in the modelequation. Thus, this render the solution of the dynamical problem cumbersome to handle as the governing equation now has variable coefficients.

Among the earliest researchers on the dynamic analysis of an elastic beam was the work of Krylor [1] who investigated the dynamic response of a simply supported beam, transversed by a constant force moving at a uniform speed. His results were obtained by using the method of expansion of eigen-functions. He assumed that the mass of the load was smaller than that of the beam. Later Timoshenko [2] used energy methods to obtain solutions in series form for simply 
supported finite beam on an elastic foundation subjected to time dependent point loads moving with uniform velocities across the beam. Kenny [3] similarly investigated the dynamic response of infinite elastic beams on elastic foundation under the influence of load moving at constant speeds. He included the effects of viscous damping in the governing differential equation. In a more recent development other researchers that considered the dynamic response of elastic structures under moving load include Oni and Omolofe [4], Oni and Awodola [5], Omolofeetal [6], Misra [7], Hsu[8], Liu and Chang [9], Achawakom and Jearisripong Kul [10].

In the above mentioned researched works, only structural members lie on the Winkler foundation model with foundation stiffness $\mathrm{K}$ are considered. However, Winkler foundation model has been criticised severely by authors [11, 12]. This is because the characteristic feature of the well-known Winkler foundation model is the discontinuous behaviour of the surface displacement beyond the load region, a more realistic elastic foundation model known as the Pasternak foundation model is considered in this paper. Thus, researchers who considered Pasternak foundation in their work are, Oni and Ogunbamike[13], Oni and Awodola [14], Oni and Jimoh [15], Oni and Jimoh [16], Jimoh and Ajoge [17], Oni and Jimoh [18], Jimoh and Ajoge [19]. To the best of authors knowledge, the more practical cases of non-uniform Rayleigh beam resting on Pasternak foundation and subjected to harmonically varying magnitude moving load has not been tackled.

This paper therefore, is concerned with the problem of the transverse motion of non-uniform Rayleigh beam resting on Pasternak foundation and subjected to harmonically vary magnitude moving load and with the consideration of the effect of rotatory inertia and axial force on the moving load.

\section{Mathematical Model}

This paper considers the dynamic behaviour of a non-uniform Rayleigh beam resting on Pasternak foundation when it is under the action of harmonically varying magnitude moving load. The beam's properties such as moment of inertia $I$ and the mass per unit length $\bar{m}$ of the beam vary along the span $\mathrm{L}$ of the beam. The deflection $V(x, t)$ of the beam resting on Pasternak foundationunder the action of a moving load is described by the fourth order partial differential equation

$$
\begin{gathered}
\frac{\partial^{2}}{\partial x^{2}}\left(E I(x) \frac{\partial^{2} V(x, t)}{\partial x^{2}}\right)+\bar{m}(x) \frac{\partial^{2} V(x, t)}{\partial t^{2}} \\
-N \frac{\partial^{2} V(x, t)}{\partial x^{2}}-R_{0}^{2} \frac{\partial}{\partial x}\left(\bar{m}(x) \frac{\partial^{3} V(x, t)}{\partial x \partial t^{2}}\right) \\
\in \frac{\partial v(x, t)}{\partial t}+K V(x, t)+G \frac{\partial^{2} V(x, t)}{\partial x^{2}} \\
=P(x, t)
\end{gathered}
$$

Where

$\bar{m}(x)=$ Variable mass per unit length of the beam

$\mathrm{I}(x)=$ variable moment of inertia

$\mathrm{N}=$ Axial force

$\epsilon=$ damping coefficient

$\mathrm{G}=$ shear modulus

$\mathrm{K}$ = foundation modulus

$R_{0}=$ Rotatory inertia

$E=$ young modulus

$V(x, t)=$ transverse deflection

$x$ is the spatial coordinate taking along the axis of the beam, and $t$ is the time.

The boundary conditions at the end $x=0$ and $x=\mathrm{L}$ are given by 


$$
V(x, t)=0=\frac{\partial V(L, t)}{\partial x}
$$

And the initial conditions as

$$
V(x, o)=0=\frac{\partial V(x, o)}{\partial x}
$$

For the variable moment of inertia $\mathrm{I}(\mathrm{x})$ and the mass per unit length $\overline{\mathrm{m}}(\mathrm{x})$ of the beam, we adopt the example in [20] and take $\mathrm{I}(\mathrm{x})$ and $\overline{\mathrm{m}}(\mathrm{x})$ to be of the form

$$
\begin{gathered}
\mathrm{I}(x)=\mathrm{I}_{0}\left(1+\sin \frac{\pi x}{L}\right)^{3} \\
\bar{m}(x)=\bar{m}_{0}\left(1+\sin \frac{\pi x}{L}\right)
\end{gathered}
$$

Furthermore, the harmonically varying magnitude moving force $P(x, t)$ acting on the beam is given by

$$
P(x, t)=P \cos \omega t \cdot f(x-c t)
$$

Where $w$ is the frequency of the load, $c$ is the velocity of the moving load and $f($.$) is the dirac-delta function.$

When equation (4), (5) (6) are substituted into equation (1), we obtain a non-homogenous partial differential equation with variable coefficient given as

$$
\begin{gathered}
E I_{0} \frac{\partial^{2}}{\partial x^{2}}\left(\left(1+\sin \frac{\pi x}{L}\right)^{3}\right) \frac{\partial^{2} V(x, t)}{\partial x^{2}}+\bar{m}_{0}\left(1+\sin \frac{\pi x}{L}\right) \frac{\partial^{2} V(x, t)}{\partial t^{2}} \\
-N \frac{\partial^{2} V(x, t)}{\partial x^{2}}-R_{0}^{2} \bar{m}_{0} \frac{\partial}{\partial x}\left(\left(1+\sin \frac{\pi x}{L}\right)\right) \frac{\partial^{3} V(x, t)}{\partial x \partial t^{2}} \\
\varepsilon \frac{\partial v(x, t)}{\partial t}+ \\
=P \operatorname{Vos}(x, t)+G \frac{\partial^{2} V(x, t)}{\partial x^{2}} \\
\end{gathered}
$$

Equation (7) can be simplified to obtain

$$
\begin{gathered}
E I_{0}\left(\frac{5}{2}+\frac{15 \sin \pi x}{4 L}-\frac{\sin 3 \pi x}{4 L}-\frac{3 \cos 2 \pi x}{2 L}\right) \frac{\partial^{4} V(x, t)}{\partial x^{4}}+E I_{0}\left(\frac{9 \pi^{2} \sin 3 \pi x}{4 L^{3}}-\frac{15 \sin \pi x}{4 L^{3}}+\frac{6 \pi^{2} \cos 2 \pi x}{L^{3}}\right) \frac{\partial^{2} V(x, t)}{\partial x^{2}} \\
+\bar{m}_{0} \frac{\partial^{2} V(x, t)}{\partial t^{2}}+\bar{m}_{0} \frac{\sin \pi x}{L} \frac{\partial^{2} V(x, t)}{\partial t^{2}}-N \frac{\partial^{2} V(x, t)}{\partial x^{2}}-R_{0}^{2} \bar{m}_{0}\left(1+\frac{\sin \pi x}{L}\right) \frac{\partial^{4} V(x, t)}{\partial x^{2} \partial t^{2}}-R_{0}^{2} \frac{\pi \bar{m}_{0}}{L} \cos \pi x \frac{\partial^{3} V(x, t)}{\partial x \partial t^{2}} \\
+\varepsilon \frac{\partial v(x, t)}{\partial t}+K V(x, t)+G \frac{\partial^{2} V(x, t)}{\partial x^{2}} \\
=M g \cos \omega t f(x-c t)
\end{gathered}
$$

\section{Appropriate Analytical Solution}

In order to solve the beam problem above, we shall use the versatile solution technique called Galerkin's method often used in solving all diverse problems involving mechanical vibrations [21,22,23]. This solution technique involves solving equations of the form 


$$
\beta(V)-P=0
$$

Where $\beta$ is the differential operator, $V$ is the structural displacement and $P$ is the transverse load acting on the structure. Thus, the solution of equation (7) is expressed as

$$
V_{j}(\mathrm{x}, \mathrm{t})=\sum_{j=1}^{n} Y_{j}(t) X_{j}(x)
$$

Where $X_{j}(x)$ is chosen to satisfy the pertinent boundary conditions in (2) putting equation (10) into (8) gives

$$
\begin{gathered}
\sum_{j=1}^{n}\left\{\frac{E I_{0}}{4}\left(10+\frac{15 \sin \pi x}{L}-\sin \frac{3 \pi x}{L}-\frac{6 \cos 2 \pi x}{L}\right) X_{j}^{i v}(x) Y_{j}(t)\right. \\
+\frac{E I_{0}}{4 L^{2}}\left(\frac{9 \pi^{2} \sin 3 \pi x}{L}-\frac{15 \sin \pi x}{L}+\frac{24 \pi x \cos 2 \pi x}{L}\right) X_{j}^{\prime \prime}(x) Y_{j}(t) \\
+\bar{m}_{0}\left(1+\frac{\sin \pi x}{L}\right) X_{j}(x) \ddot{Y}_{J}(t)-N X_{j}^{\prime \prime}(x) Y_{j}(t) \\
-R_{0}^{2} \bar{m}_{0}\left(1+\frac{\sin \pi x}{L}\right) X_{j}^{\prime \prime}(x) \ddot{Y}_{J}(t)-R_{0}^{2} \frac{\bar{m}_{0} \pi}{L} \cos \frac{\pi x}{L} X_{j}^{\prime}(x) \ddot{Y}_{J}(t) \\
+\varepsilon X_{j}(x) \dot{Y}_{j}(t)+K X_{j}(x) Y_{j}(t)+G X_{j}^{\prime \prime}(x) Y_{j}(t) \\
-M g \cos \omega t f(x-c t)\}=0
\end{gathered}
$$

In order to determine $Y_{j}(t)$,it is required that the expression on the left hand side of equation (11) be orthogonal to the function $X_{m}(x)$

Hence,

$$
\begin{gathered}
\int_{0}^{L} \sum_{j=1}^{n}\left\{\frac{E I_{0}}{4}\left(10+\frac{15 \sin \pi x}{L}-\frac{\sin 3 \pi x}{L}-\frac{6 \cos 2 \pi x}{L}\right) X_{j}^{i v}(x) Y_{j}(t)\right. \\
+\frac{E I_{o}}{4 L_{2}}\left(\frac{9 \pi^{2} \sin 3 \pi x}{L}-\frac{15 \sin \pi x}{L}-\frac{\sin 3 \pi x}{L}-\frac{6 \cos 2 \pi x}{L}\right) X_{j}^{i v}(x) Y_{j}(t) \\
+\frac{E I_{o}}{4 L_{2}}\left(\frac{9 \pi^{2} \sin 3 \pi x}{L}-\frac{15 \sin \pi x}{L}+\frac{24 \pi^{2} \cos 2 \pi x}{L}\right) X_{j}^{\prime \prime}(x) Y_{j}(t) \\
+\bar{m}_{0}\left(1+\frac{\sin \pi x}{L}\right) X_{j}(x) \ddot{Y}_{j}(t)-N X_{j}^{\prime \prime}(x) Y_{j}(t) \\
-R_{0}^{2} \bar{m}_{0}\left(1+\frac{\sin \pi x}{L}\right) X_{j}^{\prime \prime}(x) \ddot{Y}_{j}(t)-R_{0}^{2} \frac{\bar{m}_{0} \pi}{L} \cos \frac{\pi x}{L} X_{j}^{\prime}(x) \ddot{Y}_{J}(t) \\
\left.+E X_{j}(x) \dot{Y}_{J}(t)+K X_{j}(x) Y_{j}(t)+G X_{j}^{\prime \prime}(x) Y_{j}(t)\right\} X_{m}(x) d x \\
=\int_{0}^{L} M g \cos \omega t f(x-C t) X_{m}(x) d x
\end{gathered}
$$

Since, the elastic beam has been simply supported at the edges $x=0$ and $x=L$, we choose 


$$
\begin{gathered}
X_{j}(x)=\frac{\sin j \pi x}{L} \\
\text { and } X_{m}(x)=\frac{\sin m \pi x}{L}
\end{gathered}
$$

Substituting (13) and (14) into (12) after some simplification and rearrangement and ignoring the summation sign to obtain

$$
\begin{gathered}
\int_{0}^{L}\left\{\left[-R_{0}^{2} \bar{m}_{0}\left(\frac{j \pi}{L}\right)^{2}\left(\frac{\sin j \pi x}{L} \sin \frac{m \pi x}{L}+\frac{\sin \pi x}{L} \frac{\sin j \pi x}{L} \frac{\sin m \pi x}{L}\right)-R_{0}^{2} \bar{m}_{0}\left(\frac{j \pi}{L}\right)^{2} \frac{\cos \pi x}{L} \frac{\cos j \pi x}{L} \frac{\sin m \pi x}{L}\right.\right. \\
\left.+\bar{m}_{0}\left(\frac{\sin j \pi x}{L} \frac{\sin m \pi x}{L}+\frac{\sin \pi x}{L} \frac{\sin j \pi x}{L} \frac{\sin k \pi x}{L}\right)\right] \ddot{Y}_{J}(t)+\varepsilon \frac{\sin j \pi x}{L} \frac{\sin m \pi x}{L} \dot{Y}_{J}(t) \\
+\left[\frac { E I _ { 0 } } { 4 } ( \frac { j \pi } { L } ) ^ { 4 } \left(10 \frac{\sin j \pi x}{L} \frac{\sin m \pi x}{L}+\frac{15 \sin \pi x}{L} \frac{\sin j \pi x}{L} \frac{\sin m \pi x}{L}-\frac{\sin 3 \pi x}{L} \frac{\sin j \pi x}{L} \frac{\sin m \pi x}{L}-\frac{6 \cos 2 \pi x}{L} \frac{\sin j \pi x}{L} \frac{\sin m \pi x}{L}\right.\right. \\
-\frac{E I_{0}}{4 L^{2}}\left(\frac{j \pi}{L}\right)^{2}\left(\frac{9 \pi^{2} \sin 3 \pi x}{L} \frac{\sin j \pi x}{L} \frac{\sin m \pi x}{L}+\frac{15 \sin \pi x}{L} \frac{\sin j \pi x}{L} \frac{\sin m \pi x}{L}\right. \\
\left.\left.\left.+\frac{24 \pi^{2} \cos 2 \pi x}{L} \frac{\sin j \pi x}{L} \frac{\sin m \pi x}{L}\right)+N\left(\frac{j \pi^{2}}{L}\right) \frac{\sin j \pi x \sin m \pi x}{L}+K \frac{\sin j \pi x}{L} \frac{\sin m \pi x}{L}-G\left(\frac{j \pi^{2}}{L}\right) \frac{\sin j \pi x}{L} \frac{\sin m \pi x}{L}\right] Y_{j}(t)\right\} d x \\
=\int_{0}^{L} M g \cos \omega t f(x-c t) \frac{\sin m \pi x}{L} d x \\
=M g \cos w t \frac{\sin m \pi c t}{L}
\end{gathered}
$$

Equation (15) can be re-written as

$$
\wedge_{11} \ddot{Y}_{j}(t)+\Lambda_{12} \dot{Y}_{J}(t)+\wedge_{13} Y_{J}(t)=\Lambda_{14}\left(\sin A_{1}(t)-\sin A_{2}(t)\right)
$$

Where

$$
\begin{gathered}
\wedge_{11}=\left(d_{1}+\bar{m}_{0}\right)\left(C_{1}+C_{2}\right)-d_{2} C_{3} \\
\wedge_{12}=\varepsilon C_{1} \\
\wedge_{13}=d_{3}\left(\frac{5 C_{1}}{2}+\frac{15 C_{2}}{4}-\frac{C_{4}}{4}-\frac{3 C_{5}}{2}\right)-d_{4}\left(\frac{9 \pi^{2} C_{4}}{4 L^{2}}-\frac{15 C^{2}}{4 L^{2}}+\frac{6 \pi^{2} C_{5}}{L^{2}}\right)+\left(d_{5}-d_{6}+K\right) C_{1} \\
\Lambda_{14}=\frac{M g}{2 \Lambda_{11}}, \quad A_{1}=\omega+C_{0}, \quad A_{2}=\omega-C_{0} \\
C_{0}=\frac{m \pi c}{L}
\end{gathered}
$$




$$
\begin{gathered}
d_{1}=\bar{m}_{0}\left(\frac{j \pi}{L}\right)^{2} R_{0}^{2}, \quad d_{2}=\bar{m}_{0} J\left(\frac{\pi}{L}\right)^{2} R_{0}^{2}, \quad d_{3}=E I_{0}\left(\frac{j \pi}{L}\right)^{4} \\
d_{4}=E I_{0}\left(\frac{j \pi}{L}\right)^{2}, d_{5}=N\left(\frac{j \pi}{L}\right)^{2}, d_{6}=G\left(\frac{j \pi}{L}\right)^{2} \\
c_{1}=\int_{0}^{L} \frac{\sin j \pi x}{L} \frac{m \pi x}{L} d x, c_{2}=\int_{0}^{L} \frac{\sin \pi x}{L} \frac{\sin j \pi x}{L} \frac{\sin m \pi x}{L} d x \\
c_{3}=\int_{0}^{L} \frac{\cos \pi x}{L} \frac{\cos j \pi x}{L} \frac{\sin m \pi x}{L} d x, c_{4}=\int_{0}^{L} \frac{\sin 3 \pi x}{L} \frac{\sin j \pi x}{L} \frac{\sin m \pi x}{L} d x \\
c_{5}=\int_{0}^{L} \frac{\cos 2 \pi x}{L} \frac{\sin j \pi x}{L} \frac{\sin m \pi x}{L} d x
\end{gathered}
$$

By evaluating the integral in (21) for $j=m$, we obtain

$$
\begin{gathered}
c_{1}=\int_{0}^{L} \frac{\sin m \pi x}{L} \frac{\sin m \pi x}{L} d x=\frac{L}{2} \\
c_{2}=\int_{0}^{L} \frac{\sin \pi x}{L} \frac{\sin j \pi x}{L} \frac{\sin m \pi x}{L} d x=\frac{L}{4 \pi}\left[2(1-\cos \pi)+\frac{\cos (1+2 m) \pi-1}{1+2 m}+\frac{\cos (1-2 m) \pi-1}{1-2 m}\right] \\
c_{3}=\int_{0}^{L} \frac{\cos \pi x}{L} \frac{\cos m \pi x}{L} \frac{\sin m \pi x}{L} d=\frac{L}{4 \pi}\left[\frac{1-\cos (1+2 m) \pi}{1+2 m}+\frac{\cos (1-2 m) \pi-1}{1-2 m}\right] \\
c_{4}=\int_{0}^{L} \frac{\sin 3 \pi x}{L} \frac{\sin m \pi x}{L} \frac{\sin m \pi x}{L}=\frac{L}{4 \pi}\left[\frac{\cos (3+2 m) \pi-1}{3+2 m}-\frac{(\cos (3 \pi)-1)}{3 \pi}+\frac{\cos (3-2 m) \pi-1}{3-2 m}-\frac{(\cos (3 \pi)-1)}{3 \pi}\right] \\
c_{5}=\int_{0}^{L} \frac{\cos 2 \pi x}{L} \frac{\sin m \pi x}{L} \frac{\sin m \pi x}{L} d x=\frac{L}{8 \pi}\left[2 \sin (2 \pi)-\frac{\sin (1+m) 2 \pi}{1+m}+\frac{\sin (1-m) 2 \pi}{1-m}\right]
\end{gathered}
$$

Equation (16) can be re-written as

$$
\ddot{Y}_{J}(t)+\wedge_{22} \dot{Y}_{j}(t)+\wedge_{33} Y_{j}(t)=\wedge_{44}\left(\sin A_{1} t-\sin A_{2} t\right)
$$

Where

$$
\Lambda_{22}=\frac{\Lambda_{12}}{\Lambda_{11}}, \Lambda_{33}=\frac{\Lambda_{13}}{\Lambda_{11}}, \Lambda_{44}=\frac{\Lambda_{14}}{\Lambda_{11}}
$$

Equation (27) can be solved using the Laplace transformed defined by

$$
\operatorname{Le}(Y(t))=Y(s)=\int_{0}^{\infty} Y(t) e^{-s t} d t
$$

By using the transformation (29) on the equation (27) in conjunction with the initial conditions (3), upon simplific ation, we obtained

$$
Y_{j}(s)=\frac{\wedge_{44}}{\left(s-r_{1}\right)\left(s-r_{2}\right)}\left[\frac{A_{1}}{s^{2}+A_{1}^{2}}-\frac{A_{2}}{s^{2}+A_{2}^{2}}\right]
$$

Specifying (30) to obtain 


$$
Y_{j}(s)=\frac{\wedge_{44}}{r_{1}-r_{2}}\left[\begin{array}{c}
\left(\frac{1}{s-r_{1}}\right)\left(\frac{A_{1}}{s^{2}+A_{1}^{2}}\right)-\left(\frac{1}{s-r_{1}}\right)\left(\frac{A_{2}}{s^{2}+A_{2}^{2}}\right) \\
-\left(\frac{1}{s-c_{2}}\right)\left(\frac{A_{1}}{s^{2}+A_{1}^{2}}\right)+\left(\frac{1}{s-A_{2}}\right)\left(\frac{A_{2}}{s^{2}+A_{2}^{2}}\right)
\end{array}\right]
$$

Where

$$
\begin{aligned}
& r_{1}=\frac{-\Lambda_{22}}{2}+\frac{\sqrt{\Lambda_{22}^{2}-4 \Lambda_{33}}}{2} \\
& r_{2}=\frac{-\Lambda_{22}}{2}+\frac{\sqrt{\Lambda_{22}^{2}-4 \Lambda_{33}}}{2}
\end{aligned}
$$

To obtain the Laplace inversion of (31), we adopt the following representation

Substituting (33) into (31) to obtains

$$
\begin{aligned}
F_{1}(s) & =\frac{1}{s-r_{1}}, F_{2}(s)=\frac{1}{s-r_{2}} \\
G_{1}(s) & =\frac{A_{1}}{s^{2}+A_{1}^{2}}, G_{2}(s)=\frac{A_{2}}{s^{2}+A_{2}^{2}}
\end{aligned}
$$

$$
Y_{j}(s)=\frac{\wedge_{44}}{r_{1}-r_{2}}\left[F_{1}(s) G_{1}(s)+F_{2}(s) G_{2}(s)\right]
$$

To obtain the inversion Laplace of (34), we apply convolution theorem defined as

$$
\left(F_{i} * G_{i}\right)(t)=\int_{0}^{t} F_{i}(t-u) G_{i}(u) d u, i=1,2
$$

Thus, the Laplace inversion of (35) becomes

$$
Y_{j}(t)=Q\left[e^{r_{1} t} I_{1}-e^{r_{1} t} I_{2}-e^{r_{2} t} I_{3}+e^{r_{2} t} I_{4}\right]
$$

Where

$$
\begin{gathered}
Q=\frac{\wedge_{44}}{r_{1}-r_{2}} \\
I_{1}=\int_{0}^{t} e^{-r_{1} u} \sin A_{1} u d u \\
I_{2}=\int_{0}^{t} e^{-r_{1} u} \sin A_{2} u d u \\
I_{3}=\int_{0}^{t} e^{-r_{2} u} \sin A_{1} u d u \\
I_{4}=\int_{0}^{t} e^{-r_{2} u} \sin A_{2} u d u
\end{gathered}
$$

By evaluating the integrals (37-41) we obtain

$$
I_{1}=\frac{A_{1}}{A_{1}^{2}+r_{1}^{2}}\left(1-e^{-r_{1} t} \cos A_{1} t\right)-\frac{r_{1}}{A_{1}^{2}+r_{1}^{2}} e^{-r_{1} t} \sin A_{1} t
$$




$$
\begin{aligned}
& I_{2}=\frac{A_{2}}{A_{2}^{2}+r_{1}^{2}}\left(1-e^{-r_{1} t} \cos A_{2} t\right)-\frac{r_{1}}{A_{1}^{2}+r_{1}^{2}} e^{-r_{1} t} \sin A_{2} t \\
& I_{3}=\frac{A_{1}}{A_{1}^{2}+r_{2}^{2}}\left(1-e^{-r_{2} t} \cos A_{1} t\right)-\frac{r_{2}}{A_{1}^{2}+r_{2}^{2}} e^{-r_{2} t} \sin A_{1} t \\
& I_{4}=\frac{A_{2}}{A_{2}^{2}+r_{2}^{2}}\left(1-e^{-r_{2} t} \cos A_{2} t\right)-\frac{r_{2}}{A_{2}^{2}+r_{2}^{2}} e^{-r_{2} t} \sin A_{2} t
\end{aligned}
$$

Substituting (42-45) into (36) and upon simplification, we obtained

$$
\begin{gathered}
Y_{j}(t)=\frac{Q A_{1}}{A_{1}^{2}+r_{1}^{2}}\left(e^{r_{1} t}-\cos A_{1} t\right)-\frac{Q r_{1}}{A_{1}^{2}+r_{1}^{2}} \sin A_{1} t \\
-\frac{Q A_{2}}{A_{2}^{2}+r_{1}^{2}}\left(e^{r_{1} t}-\cos A_{2} t\right)+\frac{Q r_{1}}{A_{2}^{2}+r_{1}^{2}} \sin A_{2} t \\
-\frac{Q A_{1}}{A_{1}^{2}+r_{2}^{2}}\left(e^{r_{2} t}-\cos A_{1} t\right)+\frac{Q r_{2}}{A_{1}^{2}+r_{2}^{2}} \sin A_{1} t \\
+\frac{Q A_{2}}{A_{2}^{2}+r_{2}^{2}}\left(e^{r_{2} t}-\cos A_{2} t\right)-\frac{Q r_{2}}{A_{2}^{2}+r_{2}^{2}} \sin A_{2} t
\end{gathered}
$$

Putting (46) into (10) to obtain

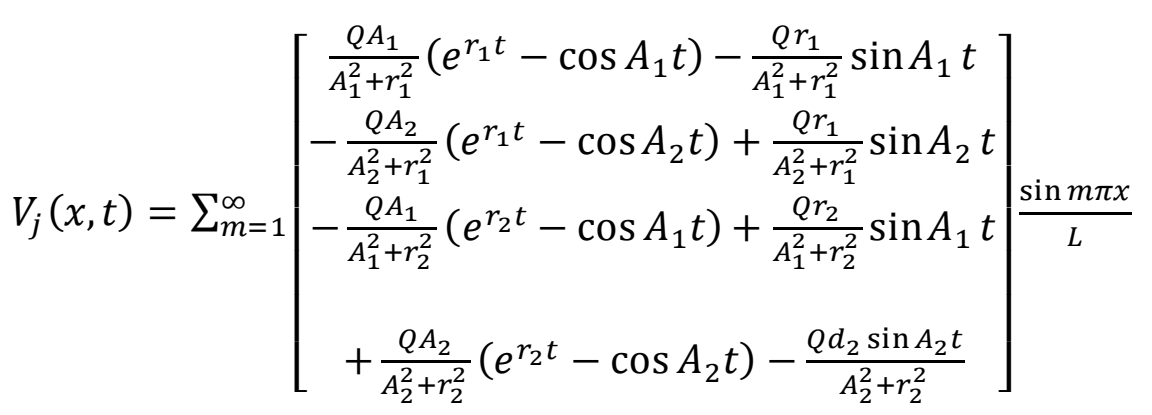

Equation (47) represents the response amplitude to harmonically varying magnitude moving loads of non-uniform Rayleigh beam resting on Pasternak foundation. 


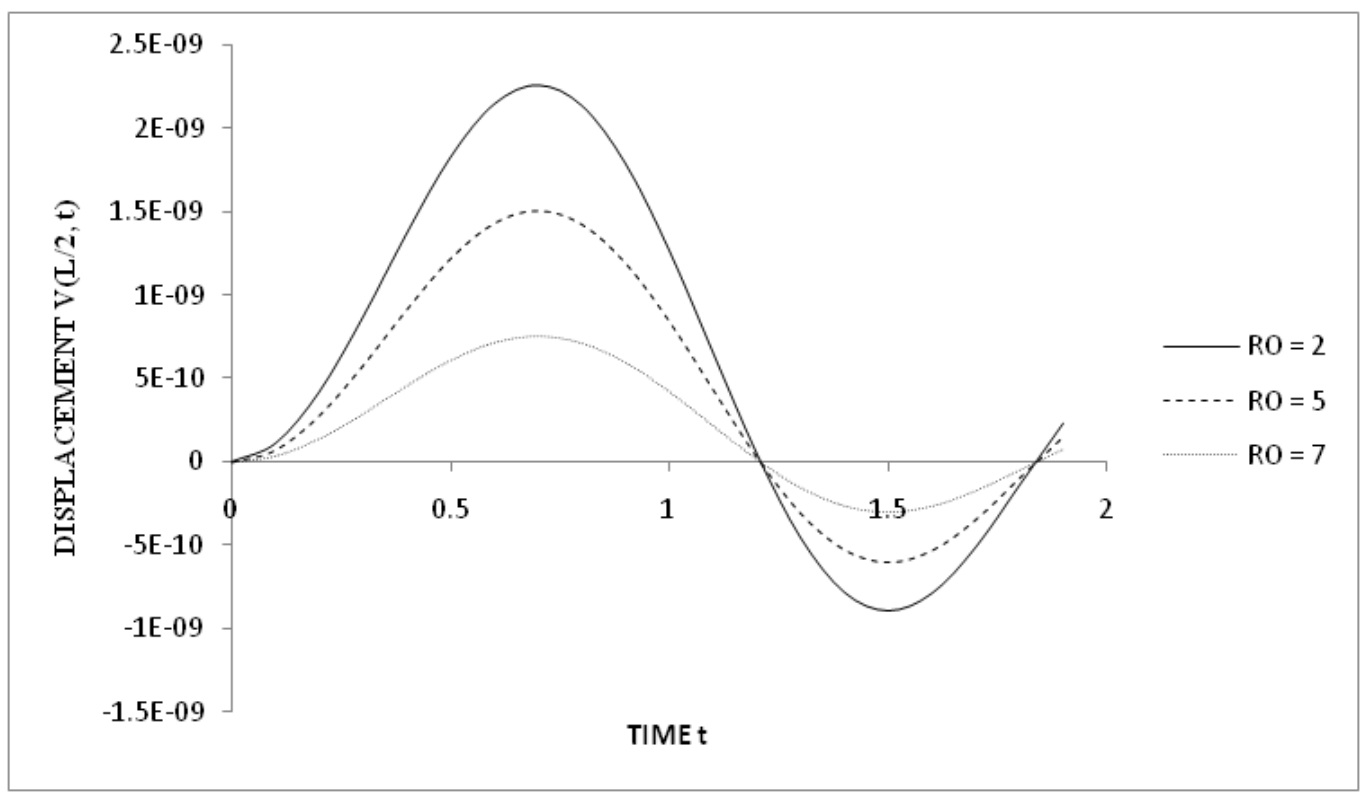

Fig 1. Deflection profile of non- uniform Raleigh beam under harmonically varying moving load for various values of Rotatory inertial R0 and fixed values of foundation modulus $\mathrm{K}$, Axial force $\mathrm{N}$, dampingcoefficient $\mathcal{E}$, shear modulus $\mathrm{G}$, load natural frequency $\omega$.

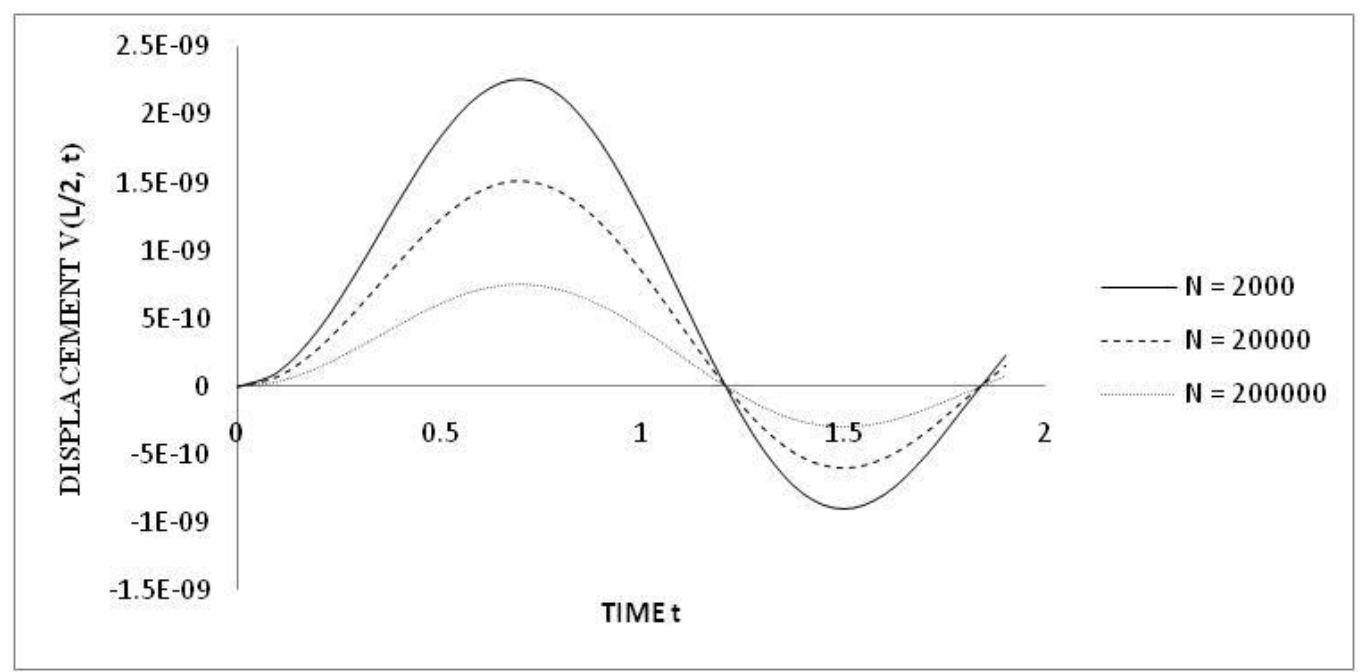

Fig 2. Deflection profile of Non- Uniform Raleigh beam under harmonically varying moving load for various values of Axial force $\mathrm{N}$, and fixed values of Rotatory inertial R0, foundation modulus $\mathrm{K}$, damping coefficient $\mathcal{E}$, shear modulus $\mathrm{G}$, and load natural frequency $\omega$. 


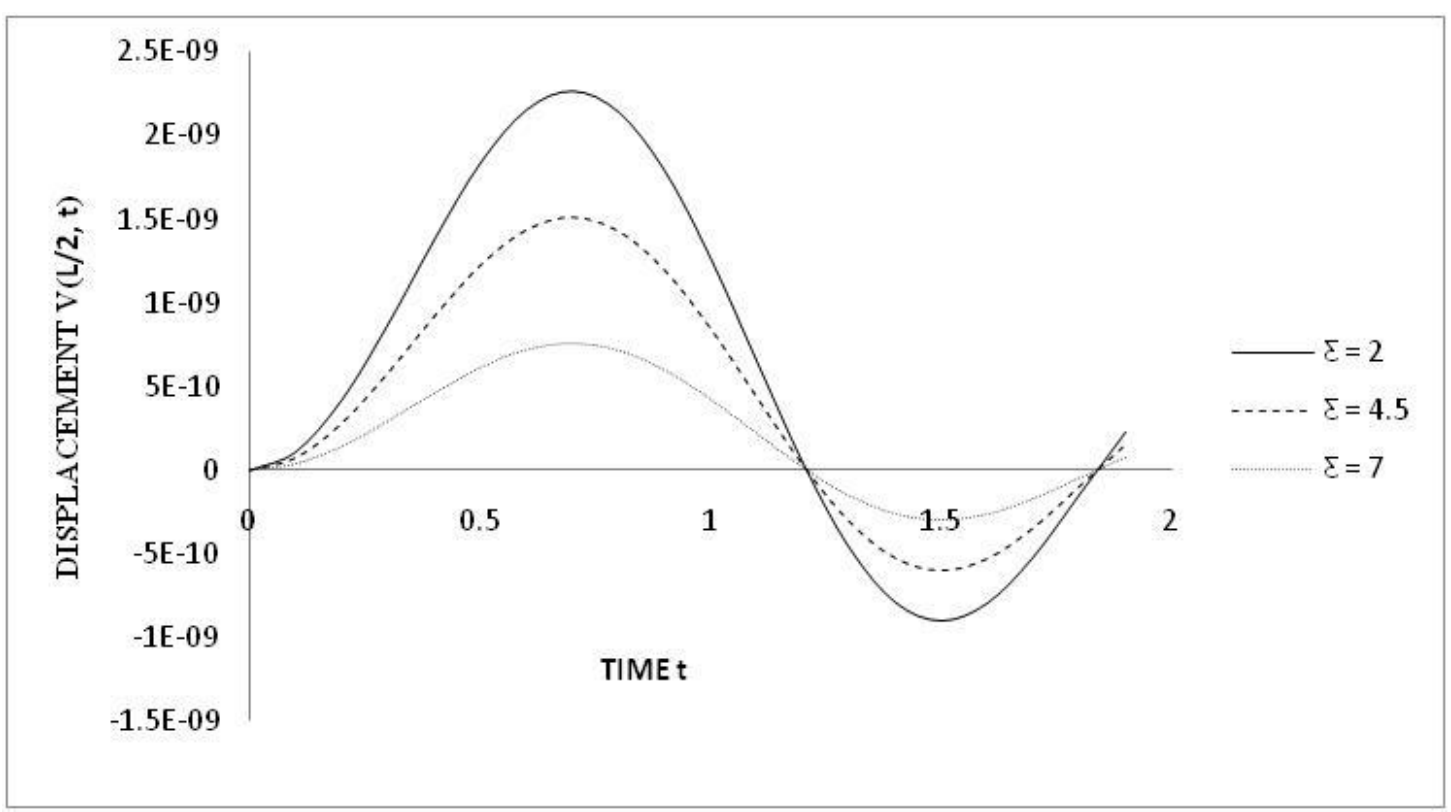

Fig 3. Deflection profile of non- uniform Raleigh beam under harmonically varying moving load for various values of damping coefficient $\Sigma$ and fixed values of Rotatory inertial $R_{o}$, foundation modulus $\mathrm{K}$, Axial force N, Shear modulus G, and load natural frequency $\omega$.

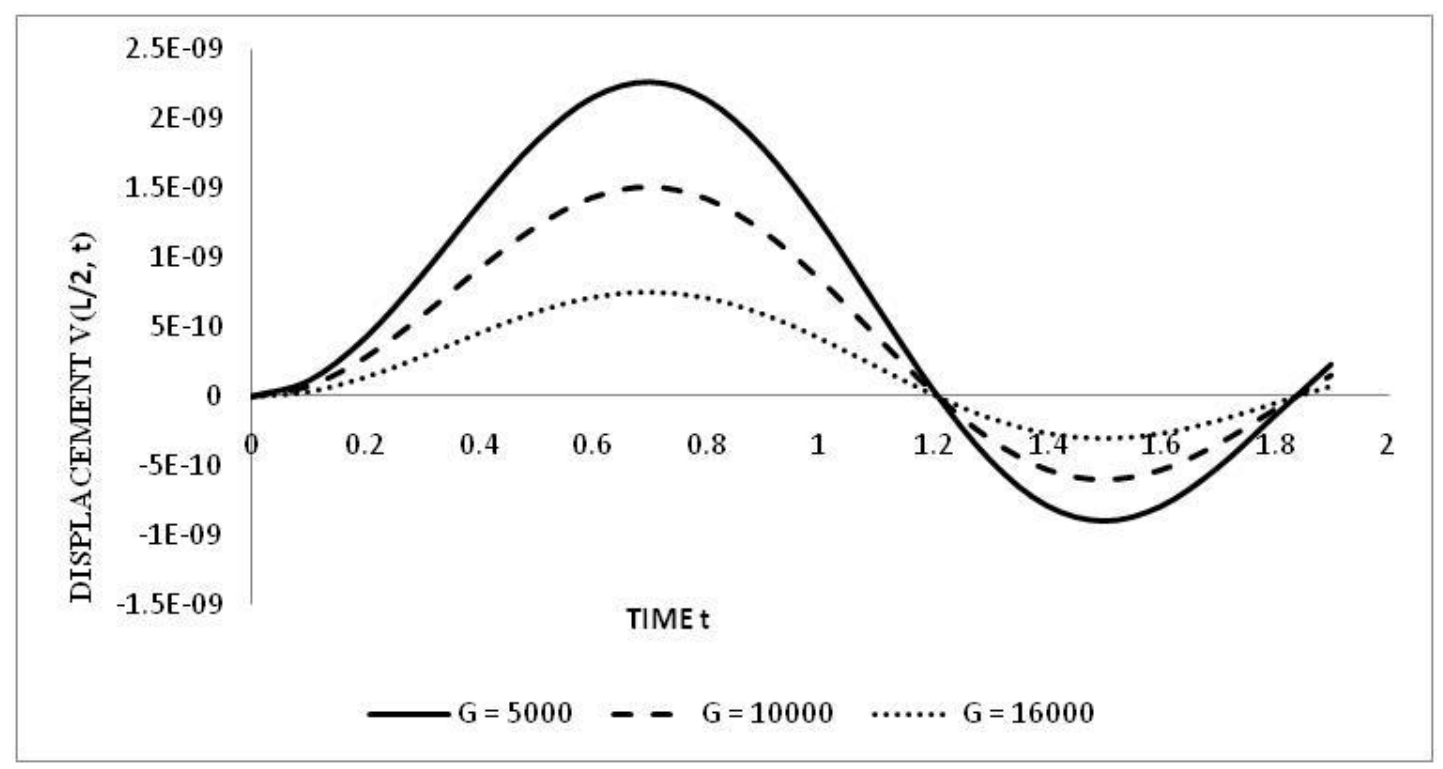

Fig 4. Deflection profile of non- uniform Raleigh beam under harmonically varying moving load for various values of Shear modulus $\mathrm{G}$,and fixed values of Rotatory inertial $R_{o}$, foundation modulus $\mathrm{K}$, Axial force $\mathrm{N}$, damping coefficient $\varepsilon$, and load natural frequency $\omega$ 


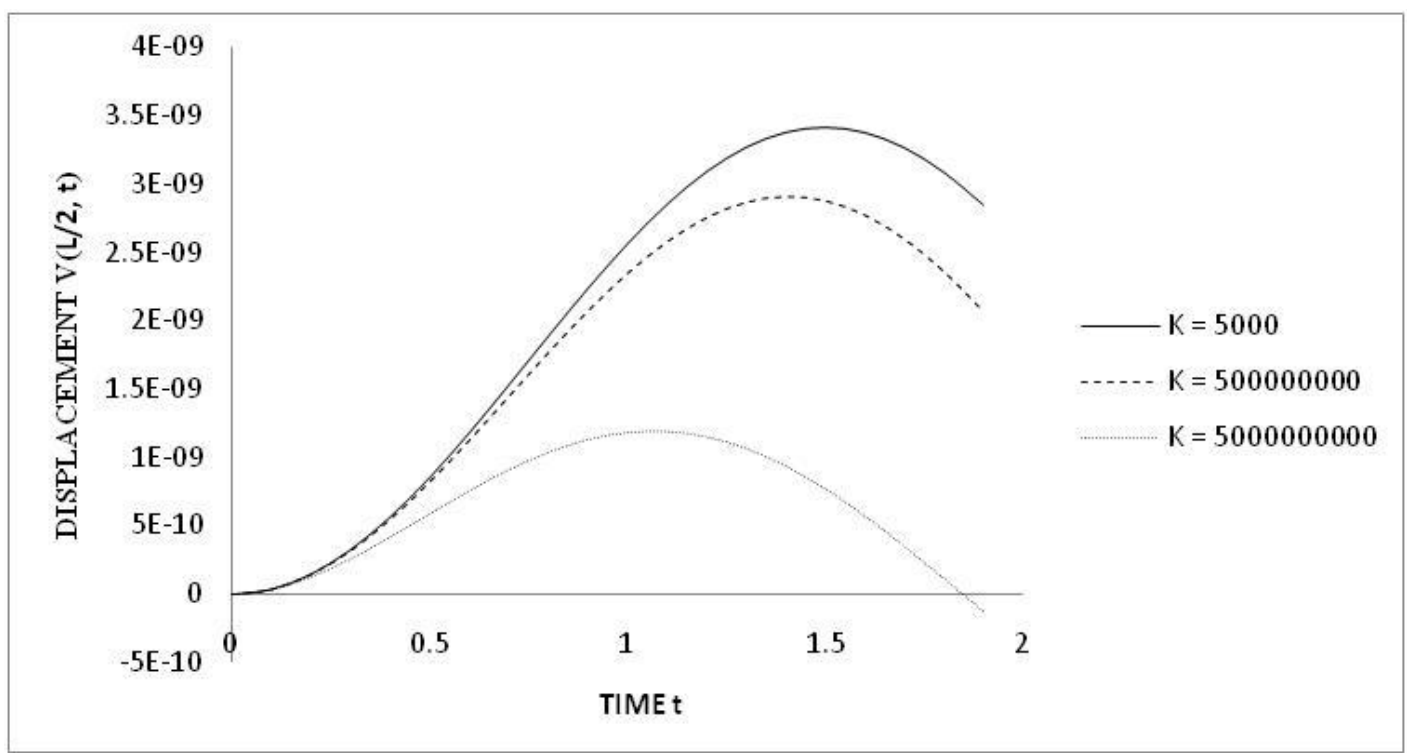

Fig 5. Deflection profile of non- uniform Raleigh beam under harmonically varying moving load for various values of foundation modulus Kand fixed values of Rotatory inertial $R_{o}$, Axial force N, damping coefficientE, Shear modulus G, and load natural frequency $\omega$.

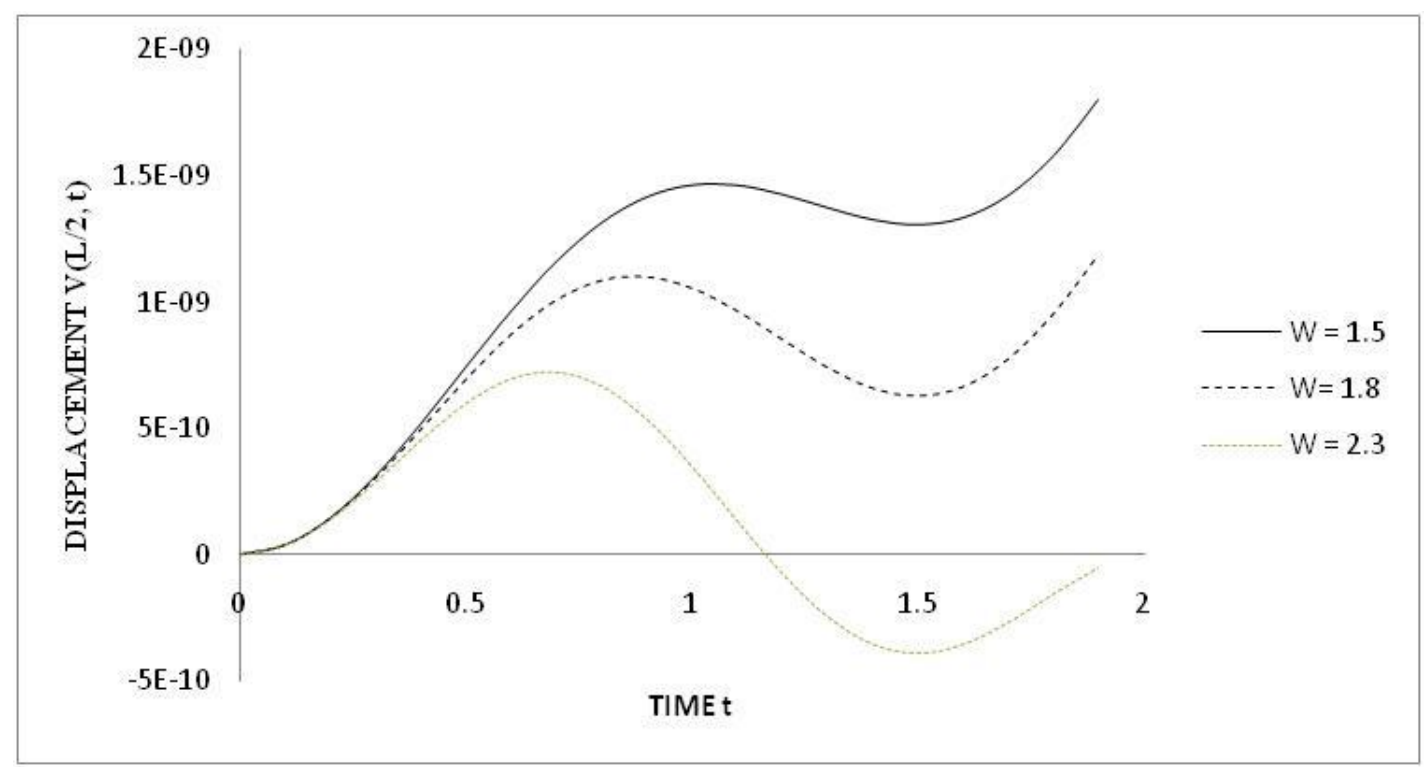

Fig 6. Deflection profile of Non- Uniform Raleigh beam under harmonically varying moving load for various values of load natural frequency $\mathrm{W}$ and fixed values of Rotatory inertial $R_{o}$, foundation modulus $\mathrm{K}$, Axial force $\mathrm{N}$, damping coefficient $\mathcal{E}$, and Shear modulus G.

\section{Numerical Analysis and Discussion of Results}

To illustrate the foregoing analysis, the non-uniform beam of length $12.192 \mathrm{~m}$, velocity of the moving load is taken to be $\mathrm{C}=8.128 \mathrm{~m} / \mathrm{s}$ are considered. The value of the foundation modulus and shear modulus are respectively varied between 
$5000 \mathrm{~N} / \mathrm{mand} 5000000 \mathrm{~N} / \mathrm{m}^{3}$ and $1000 \mathrm{~N} / \mathrm{m}^{3}$ and $10,000 \mathrm{~N} / \mathrm{m}^{3}$, values of $\varepsilon$ is between 1 and 10 , values of rotatory inertia is between 1 and 10, values of the load frequency $\omega$ is between 1 and 3 .

Figure 1 display the deflection profile of the non-uniform Rayleigh beam subjected to harmonically varying magnitude moving load. The figure shows that as the values of rotatory inertia increases, the deflection of the beam at various time $t$ decreases. Also Figure 2 also shows that an increase in the value of the axial force decreases the response amplitude of the moving load. Similarly, in Figure 3, 4, 5, \& 6 an increase in damping coefficient $(\mathcal{E})$ shear modulus $(G)$, foundation modulus $(\mathrm{K})$, and load natural frequency $\omega$ to decrease in the deflection profiles of the beam.

Finally, it was observed that the effect of rotatory inertial is more noticeable than that of the axial force.

\section{References}

[1] A.N Krylor: Mathematic collection of papers of the Academy of Sciences, Vol. 61, Piterburg 1905.

[2] S. Timoshenko: On the Correction for shear of the differential equation for transverse vibration of prismatic bars, Phil Mag. S. 6 Vol. 41. PP. 744-776, 1921

[3] J. Kenny: Steady state vibrations of a beam on an elastic foundation for a moving load. J. Appl. Mech. Vol. pp 359 -364, 1954.

[4] S.T Oni and B. Omolofe: Dynamic Analysis of a Prestressed elastic beam with general boundary condition under moving loads at varying velocities. Journal of Engineering \& Technology, FUTA. Vol. 4 No. 1 pp 55 - 72, 2005.

[5] S.T. Oni and T. Awodola: Dynamic Response to moving concentrated masses of uniform Rayleigh beams resting on variable winkler elastic foundation. Journal of the Nigerian Association of Mathematical Physics. Vol. 9, pp 151 - 162, 2005.

[6] B. Omolofe, S. T. Oni and J. M. Tolorunshagba: On the transverse motions of non-prismatic deep beam under the actions of variable magnitude moving loads. Latin American Journal of Solid and Structures. Vol 6 pp 153 - 167, 2009.

[7] R. R Misra: Free vibration analysis of isotrophical plate using multi-quadric radial basis function. International of Science, Environment and Technology. Vol. 1 (2), pp 99 - 1072012.

[8] M.H HSU: Vibration analysis of non-uniform beam resting on elastic foundation using the sine collocation method. Tankang Journal of Science and Engineering. Vol. 12 (2), pp 113 -122, 2009

[9] M. F. Liu and T. P. Chang: Closed from expression for the vibration problem of a transversely isotropic magneto -electro elastic plate. Journal of Applied Mechanic Transactions of the ASME. Vol. 77 (2) pp 025502-1-0245-2-8.

[10]K. Achawakorn and T. Jearsin-Pongjul: Vibration analsyis of exponential cross-section beam using Galerkkin's method. International Journal of Applied Sciences and Technology. Vol. 2 (6), 2010

[11]A. D. Kerr: Elastic and visco-elastic foundation models. Journal of Applied Mechanics 86, pp, 491-49, 1964

[12]G. L. Anderson: The influence of a weghardt type elastic foundation on a stability of some beams subjected to distributed tangential forces. Journal of Sound and Vibrations, 44 (1), pp $103=118,1976$.

[13] A. Jimoh and E. O. Ajoge: Influence of damping coefficient and rotatory inertia on the dynamic response to moving load of non-uniform Rayleigh beam. International Journal of Science, Engineering and Technology. pp 139 - 149, 2018

[14]S. T. Oni and A. Jimoh: On the dynamic response of moving concentrated loads of prestressed Bernoulli-Euler beam resting on Bi-parametric subgrades with other boundary conditions. Journal of Mathematical Sciences. National Mathematical Centre F.C.T Abuja. Vol. 4 No 1 pp 826 - 880. 2016.

[15] A. Jimoh and E.O Ajoge: Effect of rotatory inertial and damping coefficient on the transvers motion of uniform Rayleigh beam under moving loads of constant magnitude. American Journal of Engineering Research. Vol. 7. Issue 1, pp 313 - 319.

[16]L. Frybal: Vibrations of solids an structures under moving loads. Gromingen, Noordhoff. 1972

[17]C. Bilello and L.A. Bergman: Vibration of damaged beams under a moving mass, theory and experimental validation. Journal of Sound and Vibration, 274: $567-582,2004$.

[18]M. Dafarma, N. Jalli and E. Esmailzadeh: A comparative study of the Gaterkin approximation utilised in the Timoshenko beam theory. Journal of Sound and Vibration, 280: 1132 - 1142, 2005.

[19]E. Savin: Dynamic amplification factor and response spectrum for the evaluation of vibrations of beams under successive moving loads. Journal of Sound and Vibration, 248 (2), 267 - 288. 